\title{
Comparative study of the promotion of porcine fetal fibroblast proliferation by overexpression of two transcriptional variants of SIRT6
}

\author{
XIE XiaoXian ${ }^{1,2}$, HE Kan ${ }^{1}$, ZHANG AiLing ${ }^{2,3}$, ZHANG Hao $^{2,3}$, WANG QiShan ${ }^{1}$, \\ LI JiaQi $^{2,3} \&$ MA YuFang ${ }^{1 *}$ \\ ${ }^{1}$ School of Agriculture and Biology, Department of Animal Sciences, Shanghai Jiao Tong University, Shanghai Key Laboratory of \\ Veterinary Biotechnology, Shanghai 200240, China; \\ ${ }^{2}$ Department of Animal Genetics, Breeding and Reproduction, College of Animal Sciences, South China Agricultural University, \\ Guangzhou 510642, China; \\ ${ }^{3}$ Guangdong Provincial Key Laboratory of Agroanimal Genomics and Molecular Breeding, College of Animal Sciences, South China Agricultural \\ University, Guangzhou 510642, China
}

Received May 21, 2012; accepted September 28, 2012

\begin{abstract}
Mammalian Sirtuin 6 (SIRT6) plays an important role in the transcriptional silencing of silent mating-type loci, telomeres, and rDNA. However, the roles of porcine SIRT6 in cell proliferation are poorly understood, and a better knowledge of these will help improve our understanding of the biological mechanisms of cell growth and development. In this study, a novel variant of porcine SIRT6 (SIRT6 V2) identified by reverse transcription-polymerase chain reaction and BLAST analysis showed a 124-bp deletion compared to wild-type SIRT6 mRNA (SIRT6 V1). Two recombinant plasmids overexpressing SIRT6 V1 and SIRT6 V2 were produced and their roles in the proliferation of porcine fetal fibroblasts (PFFs) were compared. Cells transfected with SIRT6 V1 proliferated significantly faster than those transfected with SIRT6 V2 $(P<0.01)$, though both proliferated significantly faster than cells expressing an empty vector $(P<0.01)$. These results might be caused by altered proportions of $\alpha$ helices and $\beta$ sheets in the SIRT6 V2 structure relative to SIRT6 V1. These results indicate that overexpression of SIRT6 V1/V2 was involved in promoting PFF proliferation. Deletion of a 124-bp sequence attenuated the effects of SIRT6 on cell proliferation, possibly as a result of changes in the proportions of $\alpha$ helices and $\beta$ sheets in the protein secondary structure.
\end{abstract}

SIRT6 variant, overexpression, porcine fetal fibroblast, proliferation, prediction assay

Citation: Xie X X, He K, Zhang A L, et al. Comparative study of the promotion of porcine fetal fibroblast proliferation by overexpression of two transcriptional variants of SIRT6. Chin Sci Bull, 2013, 58: 1169-1174, doi: 10.1007/s11434-013-5706-5

Yeast silent information regulator 2 (SIR2) possesses $\mathrm{NAD}^{+}$-dependent deacetylase and ADP-ribosyltransferase activities, and plays essential roles in transcriptional silencing of silent mating-type loci, telomeres, and rDNA. It also represses recombination, chromosomal instability, and ageing [1-3].

SIRT6 is a distant member of the seven SIR2-derived homologs. It is expressed predominantly in nuclei and is highly correlated with heterochromatic regions. As a monoADP-ribosyltransferase, SIRT6 uses NAD ${ }^{+}$as a cosubstrate

*Corresponding author (email: mayufang@ @ sjtu.edu.cn) to transfer the ${ }^{32} \mathrm{P}$-label from $\mathrm{NAD}^{+}$to mSIRT6 and histones [4-6]. SIRT6 harbors low-level deacetylase activity for $\mathrm{NAD}^{+}$-dependent deacetylation of non-histone proteins [7] and linking site-specific histones [8-11].

SIRT6 is present in all eukaryotes including mammals [12], and deacetylates histone H3 lysine 9 (H3K9) and histone $\mathrm{H} 3$ lysine $\mathrm{K} 56$ (H3K56) to maintain dynamic changes in their acetylation levels at the telomeres over cell cycles. Histone deacetylation modifies the telomeric chromatin by preventing chromosomal fusion at the end of telomeres, and negatively regulates ageing-correlated gene-expression programs depending on nuclear factor- $\kappa \mathrm{B}[8,9,13]$. SIRT6 de- 
ficiency in mouse embryonic fibroblasts (MEFs) and embryonic cells (ES) leads to hypersensitivity to DNA damage and genomic instability with increased chromosome fragmentation, detached centromeres, and gaps, indicative of chromosomal aberrations [7]. SIRT6 deficiency also attenuates the double-strand-break repair and the base-exciserepair pathways in MEFs. SIRT6-deleted cells show increased sensitivity to DNA damage and premature senescence syndrome [7]. Inactivation of SIRT6 led to telomere dysfunction and premature senescence in human and mouse cells [9].

Deacetylation of H3K9 and H3K56 by SIRT6 decreases global histone acetylation levels in cells, mainly in $\mathrm{S}$ phase and $\mathrm{G} 2$, or during early mitosis $[8,9,13]$. Mammalian SIRT6-deficient MEFs and ES cells grew more slowly than wild-type cells in culture, and deletion of SIRT6 led to a reduced proliferation rate in several cell types $[7,14]$.

However, the mechanisms that mediate the effects of porcine SIRT6 on cell proliferation remain poorly understood. A comparison between the effects of different variants on cell proliferation will help to clarify the role of SIRT6 in the physiological regulation of cell growth and development. In this study, we identified a novel porcine SIRT6 variant and constructed plasmids to express the wild-type and novel SIRT6 variants, thus allowing the effects of both variants on porcine fetal fibroblast (PFF) proliferation to be compared.

\section{Materials and methods}

\subsection{Reverse transcription-polymerase chain reaction (PCR)}

All animal procedures were approved by the Shanghai Care and Use Committee, China. Heart, liver, spleen, lung, kidney, stomach, lymph nodes, longissimus dorsi, fat, large intestine, and cerebral tissues were harvested from Large White pigs. Total RNA was isolated from each tissue using Trizol reagent (Invitrogen, USA). cDNA was synthesized for reverse transcription-PCR from less than $5 \mu \mathrm{g}$ of each total RNA, according to the manufacturer's protocol (TaKaRa, Japan). cDNA samples $(5 \mu \mathrm{L})$ of the same concentration were pooled together to use as a template.

Porcine SIRT6 cDNA containing a complete or partial open reading frame (ORF) was cloned by reverse transcription-PCR using the primer S1 (Table 1). cDNAs containing complete or partial ORFs were named SIRT6 variant 1 (SIRT6 V1, wild-type SIRT6 mRNA) and SIRT6 variant 2 (SIRT6 V2), respectively. The reaction was carried out in a volume of $25 \mu \mathrm{L}$ for $5 \mathrm{~min}$ at $94^{\circ} \mathrm{C}, 32$ cycles of $30 \mathrm{~s}$ at $94^{\circ} \mathrm{C}, 30 \mathrm{~s}$ at $60^{\circ} \mathrm{C}$, and $40 \mathrm{~s}$ at $72^{\circ} \mathrm{C}$, ending with $8 \mathrm{~min}$ at $72^{\circ} \mathrm{C}$, with $16^{\circ} \mathrm{C}$ hold. All PCR products were purified using a MinElute Gel Extraction Kit (Qiagen, USA) and cloned into the pMD18-T vector (TaKaRa) for sequencing.

\subsection{Recombinant plasmid production for overexpres- sion of SIRT6 variants}

Two cDNA fragments of the porcine SIRT6 variants were amplified using the purified cDNA fragments as a template. The primers used were: forward primer 5'-TTTGAATTCGCTGGGAATCACCTCCGCCTT-3' (Xho I, underlined), and reverse primer 5'-TATCTCGAGGTGAGGATGTCGGTGAATTATGCG-3' (EcoR I, underlined). The fragments were cloned into the pMD18-T vector and released by restriction with Xho I and EcoR I (TaKaRa). The purified fragments were inserted into the vector pcDNA3.1(-) (Invitrogen) using T4 DNA ligase (TaKaRa) at the Xho I/EcoR I site. The constructed plasmids were named pcDNA3.1(-)-SIRT6 V1 and V2, and were identified by restriction profiling and DNA sequencing.

\subsection{Isolation, culture and transfection of PFFs}

The isolation of PFFs was performed as described previously [15]. Thawed cells were seeded into $25 \mathrm{~cm}^{2}$ flasks for expansion. Cells were cultured in Dulbecco's modified Eagle's medium (DMEM; Gibco, USA) supplemented with penicillin (100 IU/mL; Sigma, USA), streptomycin (100 $\mathrm{mg} / \mathrm{mL}$; Sigma), and 10\% fetal bovine serum (Invitrogen), and maintained at $37^{\circ} \mathrm{C}$ with $5 \% \mathrm{CO}_{2}$ humidified air. PFFs were passaged at $95 \%$ confluence. The plasmids [pcDNA3.1 (-)-SIRT6 V1/V2 and empty vector] were transfected using Lipofectamine 2000 (Sigma).

\subsection{Quantitative real-time PCR (qRT-PCR)}

Total RNA extraction and cDNA synthesis were carried out as described previously [16]. qRT-PCR was performed using SYBR Green (TaKaRa) and the ABI Prism 7000 sequence detection system (Applied Biosystems, USA). The primers S2 (Table 1) used to amplify SIRT6 V1/V2 were synthesized according to the shared coding region of both variants, with an amplicon of $248 \mathrm{bp}$. The expression of the porcine $\beta$-actin gene was evaluated as an internal control. The $\beta$-actin primer was 146 bp long (Table 1).

Table 1 The primers for amplification of SIRT6 variants cDNA and qRT-PCR

\begin{tabular}{lllc}
\hline Name & \multicolumn{1}{c}{ Primer sequences $\left(5^{\prime} \rightarrow 3^{\prime}\right)$} & $\begin{array}{c}T \mathrm{~m}^{\mathrm{a})} \\
\left({ }^{\circ} \mathrm{C}\right)\end{array}$ & $\begin{array}{c}\text { PCR } \\
\text { product } \\
\text { length }(\mathrm{bp})\end{array}$ \\
\hline S1 & $\begin{array}{l}\text { GGCGGTGAGGATGTCGGTGAA } \\
\text { S2 }\end{array}$ & 60 & $1490 / 1366$ \\
& $\begin{array}{l}\text { AAGGGGGACTGTGAGGCAATT } \\
\beta \text {-actin }\end{array}$ & & \\
& GCCATGTGGGTCTTCGTG & 58.3 & 248 \\
& AGGGCCGTGATCTCCTTCTG & & \multirow{2}{*}{146} \\
\hline
\end{tabular}

a) $\mathrm{Tm}$, optimal annealing temperature. 
The amplification reaction was initiated by pre-incubation at $95^{\circ} \mathrm{C}$ for $1 \mathrm{~min}$, followed by 40 cycles of denaturation at $95^{\circ} \mathrm{C}$ for $15 \mathrm{~s}$, annealing at $58.3^{\circ} \mathrm{C}$ for $30 \mathrm{~s}$, and extension at $72^{\circ} \mathrm{C}$ for $40 \mathrm{~s}$, performed in a $20 \mu \mathrm{L}$ volume containing $10 \mu \mathrm{L}$ SYBR Premix EX Taq GC $(2 \times), 0.4 \mu \mathrm{L}$ of each PCR primer, $0.4 \mu \mathrm{L}$ ROX reference dye $(50 \times)$, and $0.03 \mu \mathrm{g}$ cDNA. The relative expression levels were calculated using the $2^{-\Delta \Delta C_{\mathrm{T}}}$ method, as described previously [17].

\subsection{Western blot analysis}

Antibodies specific for SIRT6 were raised against the following peptide sequence: TMEERGLAPKFDTTFESARPTQTHMALVQLERVGLLRFLVSQNVDGLHVR. This sequence represents a region of human SIRT6 with 96\% similarity with the corresponding region of porcine SIRT6. Western blot analysis was performed as described previously [18]. Briefly, western blots were incubated overnight at $4^{\circ} \mathrm{C}$ with primary antibody $(1: 700$; Sigma) to porcine SIRT6, anti-glyceraldehyde phosphate 3-dehydrogenase (GAPDH) antibody (1:10000; Sigma) in phosphate-buffered saline (PBS) containing $0.05 \%$ Tween 20 with 5\% bovine serum albumin. Blots were then incubated with goat anti-rabbit horseradish peroxidase-conjugated second antibody (1:5000; Sigma) in 5\% non-fat milk for $1 \mathrm{~h}$ at room temperature. The primary antibodies were detected by antirabbit horseradish peroxidase-conjugated second antibody, using anti-GAPDH as an internal control.

\subsection{Cell counts and determination of population dou- bling times (PDs)}

The harvested cells were seeded into $75 \mathrm{~cm}^{2}$ flasks at 20000 cells per flask and cultured for $3,6,9,12$, and $15 \mathrm{~d}$, respectively. All experiments were repeated three times. The medium containing G418 was changed every $3 \mathrm{~d}$. The cells were removed at the end of each culture time and dissolved in $2 \mathrm{~mL}$ medium containing DMEM and fetal calf serum. The cells were counted using a hemocytometer. The average values were calculated using the formula: AVE = $\left(n_{1}+n_{2}+n_{3}\right) / 3$, where $n_{1}, n_{2}$, and $n_{3}$ were the counted values.

PDs were calculated according to the formula: $P D=$ $(\log N d-\log N i) / \log 2$, where $N d$ is the average number of cells counted, and $N i$ is the number of cells seeded. A growth curve was constructed according to the numbers of PDs [19]. PD analysis was used to assess the effects of both SIRT6 variants on PFFs in culture. PDs of control cells were also calculated and used as a loading control.

\subsection{Statistical analysis}

The significance of differences in cell proliferation and qRT-PCR results among cells transfected with empty vector, SIRT6 V1 and SIRT6 V2 in five separate experiments were identified by one-way analysis of variance with a Benjamini and Hochberg false discovery rate $(\mathrm{BH}-\mathrm{FDR}=0.05)$ for multiple testing correction, followed by Tukey's post-hoc tests using SPSS 10.0 software. The difference was considered significant at $P<0.05$, and extremely significant at $P<0.01$. All the data were presented as mean \pm SEM.

\subsection{Prediction of SIRT6 variant protein structures}

The deleted sequences in the SIRT6 V2 mRNA relative to SIRT6 V1 mRNA were analyzed using a multiple sequence alignment program (http://www.ebi.ac.uk/Tools/msa/ clustalw2/). The coding sequences and corresponding amino acid sequences of both SIRT6 variants were predicted using a graphical analysis tool (http://www.ncbi.nlm.nih.gov/ guide/all/) and the homology domains of both variant proteins were aligned using ClustalW. Version 2.0 of the Phyre server (http://www.sbg.bio.ic.ac.uk/servers/phyre/) was used to predict the structures of both SIRT6 proteins [20,21].

\section{Results and discussion}

\subsection{Identification and analysis of a novel variant of porcine SIRT6}

Two porcine SIRT6 variants have previously been isolated, one with a 19-bp deletion in exon 1 and a 327-bp deletion in exon 8 , and the other with a 36-bp deletion in exon 1 and a 291-bp deletion in exon 8 [22]. In this study, reverse transcription-PCR analysis of SIRT6 transcripts yielded another novel SIRT6 variant (Figure 1, not submitted to GenBank) compared to wild-type SIRT6 (GenBank accession: FJ457624) and expressed sequence tags in GenBank. This new variant was determined to have a deletion of $124 \mathrm{bp}$ using BLAST, relative to the gene sequence of porcine

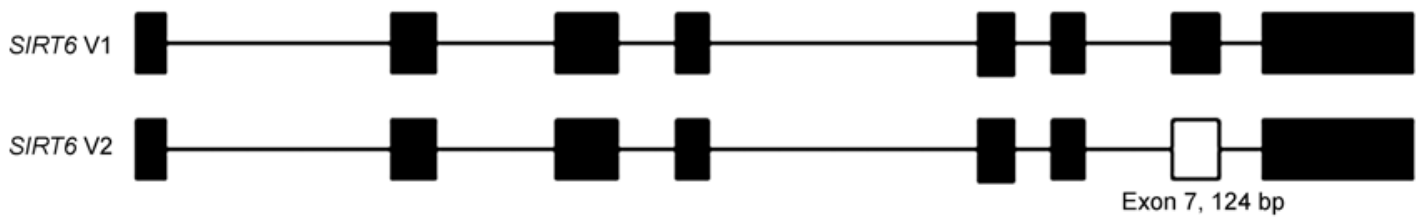

Figure 1 Identification and analysis of a novel variant of porcine SIRT6. Both SIRT6 variant mRNAs are shown relative to the gene sequence of porcine SIRT6. The exons are shaded black, except for exon 7 (124 bp), which is shaded white and was deleted in SIRT6 V2 (data not shown, GenBank accession number of the cDNA sequence was not obtained). SIRT6, Sirtuin 6; SIRT6 V1, wild-type SIRT6 mRNA; SIRT6 V2, novel variant of porcine SIRT6. 
SIRT6 (GenBank accession: HQ242712, submitted by our laboratory), which represents the complete exon 7 in SIRT6 V1.

\subsection{Construction of PFFs overexpressing SIRT6 V1 and SIRT6 V2}

cDNA sequences for SIRT6 V1 and V2 were amplified using primer S1, and cloned into the PMD18-T vector for sequencing. Fragments of 1490 and 1366 bp were subcloned into pcDNA3.1 (-) to produce pcDNA3.1(-)-SIRT6 V1 and pcDNA3.1(-)-SIRT6 V2. Both recombinant plasmids were identified with Xho I/EcoR I (Figure 2) and by DNA sequencing (data not shown).

In order to investigate the differences in cell proliferation rates between the cells transfected with SIRT6 V1 and SIRT6 V2, we generated PFF lines stably overexpressing each variant. No visible cell death or growth retardation was observed, and the cells became G418-resistant after 2 weeks using G418 at $500 \mu \mathrm{g} \mathrm{mL}^{-1}$. qRT-PCR showed upregulation of both SIRT6 variant mRNAs in these cells, to levels at least 5 -fold higher than the endogenous expression in cells transfected with empty control vector. Six overexpressing clones were selected for each of SIRT6 V1 and V2 and designated RM1-RM6 and RN1-RN6, respectively (Figure 3(a)). Clones RM1 and RN4 showed similar mRNA abundances and were used for further studies. Overexpression of both SIRT6 variants was also confirmed by Western blot analysis (Figure 3(b)). The same antibody raised against the shared region of SIRT6 V1 and SIRT6 V2 was used in the two independent experiments. The results showed more intense SIRT6 V1 and V2 protein bands in the RM1 and RN4 clones than in the control cells. SIRT6 proteins encoded by SIRT6 V1 and SIRT6 V2 hybridized to produce two bands, as seen in previous experiments $[5,11,23]$.

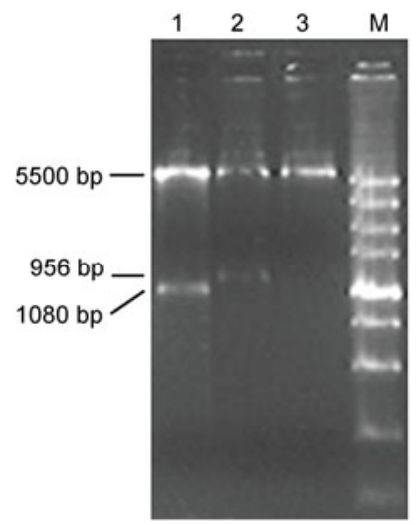

Figure 2 Identification of plasmids of both SIRT6 variants by restriction analysis. Lanes 1, 2: the recombinant plasmids pcDNA3.1(-)-SIRT6 V2 and pcDNA3.1(-)-SIRT6 V1 were restricted with Xho I/EcoR I. Fragments of 5500, 956, and $1080 \mathrm{bp}$ were produced. Lane 3: empty-vector pcDNA3.1 (-) was used as a loading control. Lane M: 5000 bp marker.
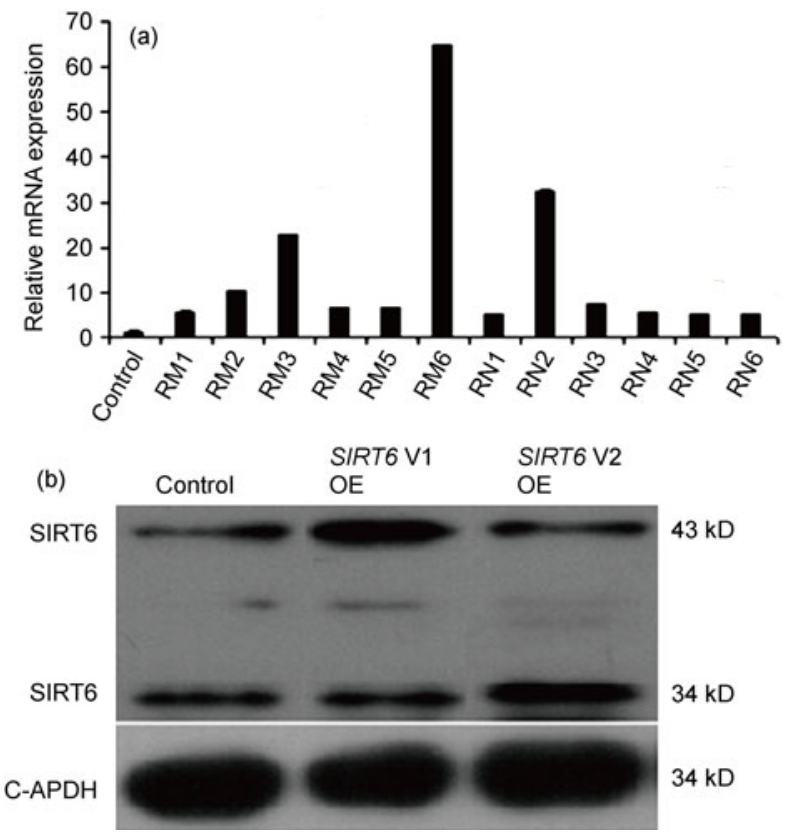

Figure 3 Construction of PFFs (porcine fetal fibroblasts) overexpressing SIRT6 V1 and V2. (a) SIRT6 mRNA expression was examined in six experimental clones transfected with SIRT6 V1 (RM1-RM6) and six transfected with SIRT6 V2 (RN1-RN6) by qRT-PCR. PFFs transfected with empty vector were examined for endogenous expression and served as a loading control. (b) Western blot assay was carried out to identify overexpression in RM1 cells transfected with SIRT6 V1 and RN4 cells transfected with SIRT6 V2. Human SIRT6 antibody was raised against the shared region of both SIRT6 variants proteins. GAPDH was used as a loading control.

\subsection{Comparison of proliferation promotion by SIRT6 V1 and SIRT6 V2}

Data were collected at different culture time points. Average cell counts at each time point were derived using the AVE formula for the cells expressing both variants and the empty vector, and PDs were calculated. A growth curve was constructed based on the various PDs (Figure 4). The PDs increased with culture time for all cell lines, and the increase was very slow for the initial $3 \mathrm{~d}$. From the third day to the end of the culture period, the rates of increase of RM1 and RN4 cells were higher than that of the control cells, with RM1 cells having the highest increase rate. Control, RM1, and RN4 cells increased by $6.65,8.61$, and 7.51 PDs, respectively. These results indicate that the cells expressing SIRT6 V1 and SIRT6 V2 proliferated faster than the control cells, by 1.96 and 0.86 PDs, respectively $(P<0.01)$. Cells transfected with SIRT6 V1 proliferated 1.1 PDs faster than cells with SIRT6 V2 over the $15 \mathrm{~d}$ culture period $(P<0.01)$.

\subsection{Prediction of SIRT6 V1 and V2 protein structures}

The coding sequences of SIRT6 V1 and SIRT6 V2 mRNAs with the initiation methionine were predicted to span 1080 and $651 \mathrm{bp}$, respectively. The corresponding amino acid sequences contained 359 and 216 amino acids (data not 


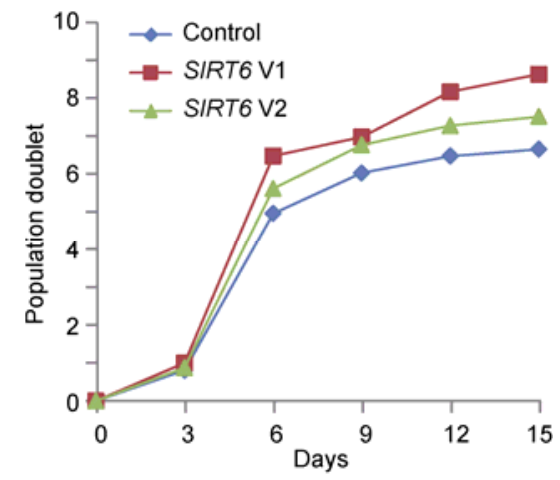

Figure 4 PDs (population doublings) determination and comparison of proliferation promotion between SIRT6 variants. PDs were determined at different culture time points, and growth curves were constructed based on number of days in culture. Cells transfected with either SIRT6 variant proliferated significantly faster than control cells $(P<0.01)$. The proliferation rate of the cells with SIRT6 V1 was significantly higher than that of the cells with SIRT6 V2 $(P<0.01)$. Growth curves were constructed for control, SIRT6 V1, and SIRT6 V2 cells, respectively.

shown). The predicted structure of SIRT6 V1 was mainly composed of $108 \alpha$ helices (31.5\%) and $89 \beta$ sheets (25.9\%), as shown in Figure 5(a), while the predicted structure of SIRT6 V2 was mainly composed of $78 \alpha$ helices $(39.0 \%)$ and $59 \beta$ sheets (29.5\%), as shown in Figure 5(b).

$\alpha$ Helices and $\beta$ sheets are the two most common types of secondary structure, and combine in different ways to create different spatial arrangements of polypeptide chains [24,25], thus playing essential roles in guiding and promoting the early steps described in many folding models [26,27]. Stabilizing mutations of the helix accelerate folding, while destabilizing mutations slow folding [28]. Destabilizing of the helix in protein $\mathrm{L}$ is the rate-limiting step in folding and unfolding, with a more significant influence on the unfolding than on the folding rate. Hydrophobic interactions and the residues in the helix are important factors in the ratelimiting step in folding [29]. A study of the mechanisms of $\beta$ sheets identified the special spatial formation as the rate-limiting step in $\beta$-sheet folding [30]. The proportions of $\alpha$ helices and $\beta$ sheets in the predicted structure of SIRT6 V2 were higher than those in the SIRT6 V1 protein (Figure $5)$, as a result of deletion of the 124-bp sequence. This might change the interactions of the secondary structures and influence their folding and unfolding rates, which in turn determine the specific tertiary structure [24,25]. In addition, all aspects of cell metabolism are catalyzed by enzymes, many of which depend on cofactors. The active configuration is produced via the combination of enzyme and cofactor, which usually includes an active site that the substrate can interact with [31]. Many enzymes are selective for just one substrate, and their activity is inhibited if the active site is blocked or the shape of the substrate is changed $[32,33]$. The change in structure of SIRT6 V2 due to the deleted 124-bp sequence (Figure 5) might thus alter the interactions between substrate and enzyme, which are involved in the series of reactions associated with cell proliferation. The altered enzyme may provide an active site that binds inhibitors more easily than substrates, or may reduce the active site thus attenuating the catalytic efficiency of the enzyme [34]. These changes might decrease the efficiency of SIRT6 variants in terms of the promotion of PFF proliferation.

In summary, overexpression of both SIRT6 variants demonstrated their involvement in the regulation of PFF proliferation. The promotion efficiency of SIRT6 V1 overexpression was significantly higher than that of SIRT6 V2 $(P<0.01)$. Deletion of a 124-bp sequence (exon 7$)$ in the ORF of SIRT6 attenuated its ability to promote PFF proliferation. It is possible that the altered proportions of $\alpha$ helices and $\beta$ sheets in the SIRT6 V2 protein structure, relative

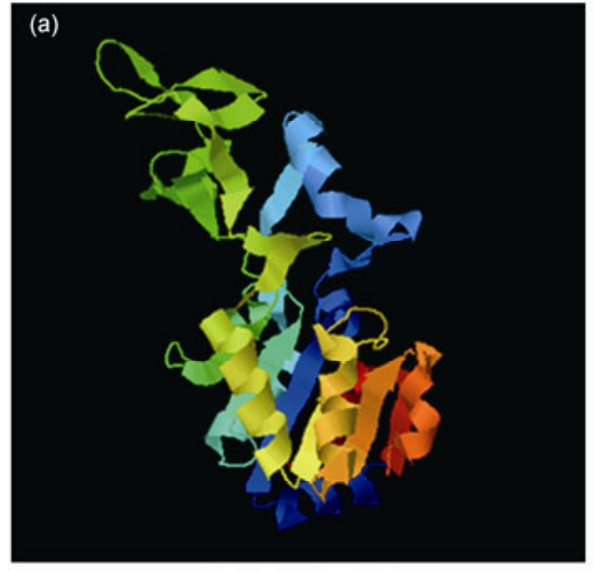

Predicted SIRT6 V1

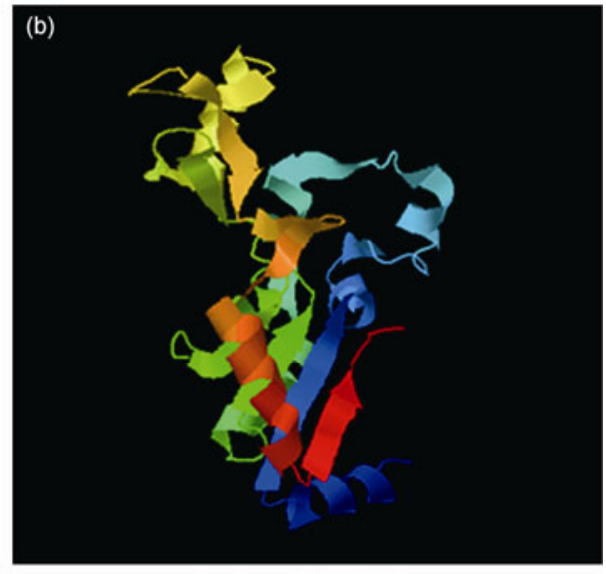

Predicted SIRT6 V2

Figure 5 Comparison of predicted SIRT6 V1 and SIRT6 V2 protein structures. The protein structures of SIRT6 V1 and V2 were predicted and compared. (a) Predicted structure of SIRT6 V1 was composed mainly of $108 \alpha$ helices and $89 \beta$ sheets, which comprised $31.5 \%$ and $25.9 \%$ of the polypeptide, respectively. (b) Predicted structure of SIRT6 V2 was composed mainly of $78 \alpha$ helices and $59 \beta$ sheets, comprising $39.0 \%$ and $29.5 \%$ of the polypeptide, respectively. 
to SIRT6 V1, may have changed their interactions and altered the folding and unfolding rates, or attenuated the catalytic efficiency of the enzymes associated with cell proliferation.

The authors wish to express their gratitude to the members of the Veterinary Biotechnology Laboratory of Shanghai Jiao Tong University, and the Agroanimal Genomics and Molecular Breeding Laboratory of South China Agricultural University. This work was supported by the National Special Fundation for Transgenic Species of China (2011ZX08006-004), and the Earmarked Fund for Modern Agro-industry Technology Research System (CARS-36).

1 Landry J, Sutton A, Tafrov S T, et al. The silencing protein SIR2 and its homologs are NAD-dependent protein deacetylases. Proc Natl Acad Sci USA, 2000, 97: 5807-5811

2 Kaeberlein M, McVey M, Guarente L. The SIR2/3/4 complex and SIR2 alone promote longevity in Saccharomyces cerevisiae by two different mechanisms. Genes Dev, 1999, 13: 2570-2580

3 Moazed D. Enzymatic activities of Sir2 and chromatin silencing. Curr Opin Cell Biol, 2001, 13: 232-238

4 Liszt G, Ford E, Kurtev M, et al. Mouse Sir2 homolog SIRT6 is a nuclear ADP-ribosyltransferase. J Biol Chem, 2005, 280: 21313-21320

5 Michishita E, Park J Y, Burneskis J M, et al. Evolutionarily conserved and nonconserved cellular localizations and functions of human SIRT proteins. Mol Biol Cell, 2005, 16: 4623-4635

$6 \mathrm{Du} \mathrm{J}$, Jiang $\mathrm{H}$, Lin $\mathrm{H}$. Investigating the ADP-ribosyltransferase activity of sirtuins with nad analogues and 32P-NAD. Biochemistry, 2009, 48: 2878-2890

7 Mostoslavsky R, Chua K F, Lombard D B, et al. Genomic instability and ageing-like phenotype in the absence of mammalian SIRT6. Cell, 2006, 124: 315-329

8 Kawahara T L, Michishita E, Adler A S, et al. SIRT6 links histone H3 lysine 9 deacetylation to NF-kappaB-dependent gene expression and organismal life span. Cell, 2009, 136: 62-74

9 Michishita E, McCord R A, Berber E, et al. SIRT6 is a histone H3 lysine 9 deacetylase that modulates telomeric chromatin. Nature, 2008, 452: 492-496

10 Zhong L, D'Urso A, Toiber D, et al. The histone deacetylase Sirt6 regulates glucose homeostasis via Hif1alpha. Cell, 2010, 140: 280293

11 Tennen R I, Berber E, Chua K F. Functional dissection of SIRT6: Identification of domains that regulate histone deacetylase activity and chromatin localization. Mech Ageing Dev, 2010, 131: 185-192

12 Frye R A. Characterization of five human cDNAs with homology to the yeast SIR2 gene: Sir2-like proteins (sirtuins) metabolize NAD and may have protein ADP-ribosyltransferase activity. Biochem Biophys Res Commun, 1999, 260: 273-279

13 Michishita E, McCord R A, Boxer L D, et al. Cell cycle-dependent deacetylation of telomeric histone H3 lysine K56 by human SIRT6.
Cell Cycle, 2009, 8: 2664-2666

14 Lombard D B, Schwer B, Alt F W, et al. SIRT6 in DNA repair, metabolism and ageing. J Intern Med, 2008, 263: 128-141

15 Boquest A C, Day B N, Prather R S. Flow cytometric cell cycle analysis of cultured porcine fetal fibroblast cells. Biol Reprod, 1999, 60: 1013-1019

16 Wang M H, Long M, Zhu B Y, et al. Effects of sargentgloryvine stem extracts on HepG-2 cells in vitro and in vivo. World J Gastroentero, 2011, 17: 2848

17 Schmittgen T D, Livak K J. Analyzing real-time PCR data by the comparative CT method. Nat Protoc, 2008, 3: 1101-1108

18 Trog D, Moenkemann H, Haertel N, et al. Expression of ABC-1 transporter is elevated in human glioma cells under irradiation and temozolomide treatment. Amino Acids, 2005, 28: 213-219

19 Blander G, Bhimavarapu A, Mammone T, et al. SIRT1 promotes differentiation of normal human keratinocytes. J Mol Biol, 2008, 129: 41-49

20 Kelley L A, Sternberg M. Protein structure prediction on the Web: A case study using the Phyre server. Nat Protoc, 2009, 4: 363-371

21 Garnier J, Osguthorpe D, Robson B. Analysis of the accuracy and implications of simple methods for predicting the secondary structure of globular proteins. J Mol Biol, 1978, 120: 97-120

22 Jin D, Tan H J, Lei T, et al. Molecular cloning and characterization of porcine sirtuin genes. Comp Biochem Physiol B Biochem Mol Biol, 2009, 153: 348-358

23 Kanfi Y, Shalman R, Peshti V, et al. Regulation of SIRT6 protein levels by nutrient availability. FEBS Lett, 2008, 582: 543-548

24 Branden C, Tooze J. Introduction to Protein Structure. New York: Garland Pub. Inc., 1998

25 Berman H M, Goodsell D S, Bourne P. Protein structures: From famine to feast. Am Sci, 2002, 90: 350

26 Kim P S, Baldwin R L. Intermediates in the folding reactions of small proteins. Annu Rev Biochem, 1990, 59: 631-660

27 Karplus M, Weaver D L. Protein folding dynamics: The diffusioncollision model and experimental data. Protein Sci, 1994, 3: 650-668

28 Sosnick T R, Jackson S, Wilk R R, et al. The role of helix formation in the folding of a fully alpha-helical coiled coil. Proteins, 1996, 24: $427-432$

29 Kim D E, Yi Q, Gladwin S T, et al. The single helix in protein L is largely disrupted at the rate-limiting step in folding. J Mol Biol, 1998, 284: 807-815

30 Deechongkit S, Nguyen H, Jager $\mathrm{M}$, et al. Beta-Sheet folding mechanisms from perturbation energetics. Curr Opin Struc Biol, 2006, 16: $94-101$

31 Zimmerman J. Enzyme kinetics and mechanism. Biochem Mol Biol Edu, 2007, 35: 386-388

32 Rabinovich M, Melnick M, Bolobova A. The structure and mechanism of action of cellulolytic enzymes. Biochem (Moscow), 2002, 67: 850-871

33 Page M, Di Cera E. Serine peptidases: Classification, structure and function. Cell Mol Life Sci, 2008, 65: 1220-1236

34 Price N C. What is meant by 'competitive inhibition'? Trends Biochem Sci, 1979, 4: N272-N273

Open Access This article is distributed under the terms of the Creative Commons Attribution License which permits any use, distribution, and reproduction in any medium, provided the original author(s) and source are credited. 